\title{
Aspectos psicossociais de acompanhantes cuidadores de crianças com câncer: stress e enfrentamento'
}

\author{
Psychosocial aspects of caregivers of children \\ with cancer: stress and coping
}

\author{
Ana Maria DEL BIANCO FARIA² \\ Carmen Lúcia CARDOSO²
}

\begin{abstract}
Resumo
O objetivo deste estudo foi avaliar o nível de stress dos cuidadores de crianças com leucemia, suas estratégias de enfrentamento, bem como caracterizar a vivência afetiva frente ao adoecimento. Participaram vinte cuidadores de crianças, após um ano de tratamento. Utilizaram-se os instrumentos: Inventário de Sintomas de Stress para Adultos de Lipp, Inventário de Estratégias de Coping e um roteiro complementar. Encontrou-se stress em 50\% dos participantes, predominância na fase de resistência e sintomatologia psicológica. Renda superior esteve associada a manifestações de stress $(p=0,02)$. As estratégias de enfrentamento mais utilizadas foram: "resolução de problemas" e "fuga e esquiva". Maior utilização da estratégia de enfrentamento "aceitação de responsabilidade" associou-se à presença de stress $(p=0,04)$ e não ser praticante de religião $(p=0,003)$. A análise da entrevista possibilitou o agrupamento em quatro categorias. Conclui-se que o cuidador necessita de atenção, visando melhorar a qualidade de vida e um maior acolhimento à criança.
\end{abstract}

Unitermos: Cuidadores. Neoplasias. Stress.

\begin{abstract}
The purpose of this study was to assess the level of stress experienced by caregivers of children with leukemia, the coping strategies they use and to characterize the affective experience towards falling ill. Twenty caregivers participated in the study, which took place one year after the children began treatment. The following instruments were used: Lipp's Adult Stress Symptoms Inventory, Coping Strategy Inventory, and a supplementary script. It was found that 50\% of the participants experienced stress, most were in the resistance phase and showed psychological symptoms. Higher income was associated with stress $(p=0.02)$. The major coping strategies used were "problem solving" and "escape and avoidance". A greater use of the "acceptance of responsibility" strategy was associated with the presence of stress $(p=0.04)$ and not practicing a religion $(p=0.003)$. The analysis of the interviews enabled a grouping into four categories. In conclusion, the caregiver needs support with a view to improving his/her quality oflife and to making children feel more welcome.
\end{abstract}

Uniterms: Caregivers. Stress. Neoplasms.

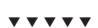

1 Apoio da Fundação de Amparo à Pesquisa do Estado de São Paulo (Processo n 2006/01165-0).

2 Universidade de São Paulo, Faculdade de Filosofia, Ciências e Letras de Ribeirão Preto, Departamento de Psicologia e Educação, Programa de Pós-Graduação em Psicologia. Av. Bandeirantes, 3900, 14040-901, Ribeirão Preto, SP, Brasil. Correspondência para/Correspondence to: C.L. CARDOSO. E-mail: <carmen@ffclrp.usp.br>. 
Câncer infantil é a denominação para um grupo de doenças que têm em comum a proliferação descontrolada de células anormais e que podem ocorrer em qualquer local do organismo. As neoplasias mais frequentes na infância são as leucemias (glóbulos brancos), tumores do sistema nervoso central e linfomas (sistema linfático) (Brasil, 2008).

O Brasil (2008) aponta que $70 \%$ das crianças acometidas pela doença podem ser curadas. Assim, a mudança na caracterização do câncer como uma doença crônica e não mais aguda e fatal trouxe a necessidade de novos enfoques, levando em conta a dimensão psicossocial da doença, e uma revisão e transformação nas práticas de saúde (Valle \& Françoso, 1999).

A ocorrência do câncer interfere de forma intensa e rápida em aspectos fundamentais da vida da criança que, diante da sensação de perigo iminente, tem a continuidade de seu desenvolvimento subitamente interrompida (Menezes, Passareli, Drude, Santos \& Valle, 2007). E, apesar do grande avanço tecnológico, que possibilita precoce prevenção, detecção e tratamento, o câncer ainda é entendido pela sociedade como uma doença incurável, com risco iminente de morte (Ulisses de Carvalho, 2008; Yin \& Twinn, 2004). Knafl e Deatrick (2003) apontam que o conjunto de crenças que os pais possuem acerca da doença e de seu curso está relacionado à capacidade das mães de lidar com o cuidado do filho. Um diagnóstico desse na infância explode de forma devastadora para a criança e para a família, afetando sua unidade e expondo-os a grandes aflições e angústias (Patterson, Holm \& Gurney, 2004). Segundo Françoso e Valle (1999), os familiares e a criança são acometidos por medo e stress durante todo o processo de diagnóstico e tratamento da criança.

Selye (1956) definiu o stress como uma quebra da homeostase interna. Segundo o autor, a reação de stress é eliciada por um estressor que consiste em qualquer evento ou situação do ambiente que quebre a homeostase interna, exigindo um esforço de adaptação. Lipp (1996) aponta que o evento em si, percebido pelos órgãos sensoriais, é interpretado de acordo com a história de vida do ser humano.

Selye (1956) descreveu três fases das alterações físicas e químicas produzidas pelo stress: fase de alerta, fase de resistência e fase de exaustão. Segundo Selye (1983), a adaptabilidade do corpo é finita; assim, nesta última fase, o organismo caminha tanto para a exaustão física quanto psicológica. Lipp e Malagris (1998) apontam que o nível de qualidade de vida encontra-se profundamente influenciado pelo nível de stress que a pessoa apresenta.

A ocorrência de manifestações de stress implica desenvolver possibilidades de lidar com as situações estressantes; nesse contexto, Lazarus e Folkman (1984) apontam que o enfrentamento (coping) do stress dá-se através de esforços cognitivos e comportamentais empreendidos para o controle de demandas específicas, ou seja, o enfrentamento é uma resposta ao stress com a finalidade de reduzir situações avaliadas como estressantes ou excessivas para o indivíduo.

Patiño-Fernández et al. (2008), em um estudo com mães e pais de crianças recentemente diagnosticadas com câncer, apontaram a presença de sintomas de stress agudo na maioria dos pais. Outros estudos, dentro da mesma temática, apontam altos níveis de manifestações de sintomas de stress pós-traumático em pais de crianças ainda em tratamento do câncer (Kazak, Boeving, Alderfer, Hwang \& Reilly, 2005) e, também, nos que já tiveram o tratamento do filho encerrado e a criança sobreviveu à doença (Kazak et al., 2004).

Nesse sentido, a cura não deve se basear somente na recuperação biológica, mas também no investimento da recuperação do bem-estar da criança, abalado anteriormente pela ocorrência da doença (Françoso \& Valle, 1999). Torna-se necessário ter atenção e cuidado a esses familiares, o que poderá favorecer a recuperação de seu bem-estar, beneficiando inclusive a criança doente.

Neste contexto, o objetivo deste estudo foi avaliar o nível de stress dos acompanhantes cuidadores de crianças com leucemia e suas estratégias de enfrentamento, bem como caracterizar a vivência afetiva desses cuidadores frente à situação de adoecimento da criança, após um ano de tratamento da doença.

\section{Método}

\section{Participantes}

O trabalho foi realizado com vinte acompanhantes cuidadores de crianças com leucemia. As crianças tinham idades entre 0 e 12 anos e estavam 
submetidas há mais de um ano de tratamento no Hospital das Clínicas da Faculdade de Medicina de Ribeirão Preto da Universidade de São Paulo (HCFMRPUSP). O perfil sociodemográfico dos participantes encontra-se na Tabela 1.

\section{Instrumentos}

- Inventário de Sintomas de Stress para Adultos de Lipp (ISSL), validado por Lipp (2000), apresenta uma avaliação de sintomas de stress, o tipo de sintomatologia e a fase em que o sujeito se encontra: alerta, resistência, quase exaustão e exaustão.

- Inventário de Estratégias de Coping de Lazarus e Folkman (IEC), validado para o Brasil por Savoia (1999) com o intuito de avaliar as estratégias de enfrentamento utilizadas para lidar com eventos estressantes específicos. Contém 66 itens que abordam oito fatores: "confronto", "afastamento", "autocontrole", "suporte so-

Tabela 1. Caracterização em frequência simples ( $f$ ) e porcentagem (\%) dos participantes $(n=20)$ de acordo com as variáveis sociodemográficas. Ribeirão Preto (SP), 2007.

\begin{tabular}{|c|c|c|c|}
\hline Variável & Especificação & $f$ & $\%$ \\
\hline \multirow[t]{2}{*}{ Sexo } & Feminino & 18 & 90,0 \\
\hline & Masculino & 2 & 10,0 \\
\hline \multirow[t]{3}{*}{ Faixa etária em anos } & $20-30$ anos & 8 & 40,0 \\
\hline & $31-40$ anos & 8 & 40,0 \\
\hline & $41-50$ anos & 4 & 20,0 \\
\hline \multirow[t]{2}{*}{ Nível educacional } & $\leq 8$ anos de estudo & 11 & 55,0 \\
\hline & $>8$ anos de estudo & 9 & 45,0 \\
\hline \multirow[t]{3}{*}{ Estado civil } & Solteiro & 2 & 10,0 \\
\hline & Casado & 14 & 70,0 \\
\hline & Separado/divorciado & 4 & 20,0 \\
\hline \multirow{3}{*}{$\begin{array}{l}\text { Parentesco com o } \\
\text { paciente }\end{array}$} & Mãe & 17 & 85,0 \\
\hline & Pai & 2 & 10,0 \\
\hline & Tia (irmã da mãe) & 1 & 5,0 \\
\hline \multirow[t]{3}{*}{ Religião } & Católica & 13 & 65,0 \\
\hline & Evangélica & 5 & 25,0 \\
\hline & Não tem & 2 & 10,0 \\
\hline \multirow[t]{2}{*}{ Praticante } & $\operatorname{sim}$ & 12 & 60,0 \\
\hline & Não & 6 & 40,0 \\
\hline \multirow{6}{*}{$\begin{array}{l}\text { Faixas de renda familiar } \\
\text { em salário-mínimo* }\end{array}$} & Sem rendimento & 0 & - \\
\hline & Até 1 salário-mínimo & 3 & 15,0 \\
\hline & Mais de 1 a 2 & 10 & 50,0 \\
\hline & Mais de 2 a 3 & 4 & 20,0 \\
\hline & Mais de 3 a 5 & 2 & 10,0 \\
\hline & Mais de 5 a 10 & 1 & 5,0 \\
\hline
\end{tabular}

* Salário-mínimo vigente na época da pesquisa $=R \$ 350,00$. cial", "aceitação de responsabilidade", "fuga e esquiva", "resolução de problemas" e "reavaliação positiva".

- Roteiro Complementar, trata-se de uma entrevista semiestruturada desenvolvida pela pesquisadora, composta de duas partes: a primeira visando levantar dados sociodemográficos e, a segunda, composta por questões tentando abarcar a vivência afetiva do acompanhante no processo de adoecimento da criança.

O estudo aqui descrito foi desenvolvido junto ao Ambulatório de Oncologia Pediátrica do Departamento de Puericultura e Pediatria do HCFMRP-USP, após a aprovação no Comitê de Ética (processo HCRP $n^{\circ}$ 2752/2006). Todos os participantes foram entrevistados face a face, individualmente, em uma única sessão, no próprio serviço, com duração de aproximadamente 50 minutos.

Procedeu-se ao tratamento estatístico não paramétrico dos dados, comparando as variáveis demográficas em relação aos indicadores de stress e os fatores de enfrentamento. Para a comparação foi utilizado o Teste de Mann-Whitney e adotaram-se valores de $p \leq 0,05$. Em relação às entrevistas, procedeu-se à Análise de Conteúdo (Bardin, 1979). Com base na transcrição literal das entrevistas foram efetuadas leituras do material, com posterior categorização das respostas, levando em conta a frequência das mesmas.

\section{Resultados}

Os resultados obtidos pelo Inventário de Sintomas de Stress para Adultos de Lipp (Lipp, 2000) apontaram que 50,0\% dos participantes apresentaram indicadores de presença de manifestações de stress. Entre os que apresentaram stress ( $n=10), 60,0 \%$ encontravam-se na fase de resistência, 30,0\% na fase de exaustão e 10,0\% na fase de quase-exaustão, o que indica a presença de stress já instalado. Cabe destacar que a sintomatologia é 90,0\% de ordem psicológica.

Em relação às estratégias de enfrentamento frente à situação de adoecimento do paciente, foi utilizada a proposta de Guido (2003) para a cotação do Inventário de Estratégias de Coping. Os resultados obtidos encontram-se na Tabela 2.

Observa-se, com base na distribuição das médias (M) das respostas dos cuidadores, que os fatores 
Tabela 2. Inventário de Estratégias de Coping de Folkman e Lazarus: mediana, média, desvio-padrão e intervalo mínimo máximo dos escores obtidos em cada um dos fatores pelos participantes. Ribeirão Preto (SP), 2007.

\begin{tabular}{|c|c|c|c|c|}
\hline $\begin{array}{l}\text { Estratégias de } \\
\text { enfrentamento } \\
\text { (Fatores do } \\
\text { inventário) }\end{array}$ & $\mathrm{Me}$ & M & DP & $\begin{array}{l}\text { Intervalo } \\
\text { mínimo- } \\
\text {-máximo }\end{array}$ \\
\hline Confronto & 0,75 & 0,81 & 0,48 & $0,00-1,83$ \\
\hline Afastamento & 0,64 & 0,67 & 0,39 & $0,00-1,42$ \\
\hline Autocontrole & 1,00 & 1,21 & 0,58 & $0,40-2,80$ \\
\hline Suporte social & 2,08 & 1,89 & 0,68 & $0,33-2,66$ \\
\hline $\begin{array}{l}\text { Aceitação de } \\
\text { responsabilidade }\end{array}$ & 0,85 & 0,92 & 0,53 & $0,14-2,00$ \\
\hline Fuga e esquiva & 2,00 & 2,05 & 0,75 & $0,50-3,00$ \\
\hline Resolução de problemas & 2,25 & 2,06 & 0,69 & $0,50-3,00$ \\
\hline Reavaliação positiva & 2,00 & 1,95 & 0,51 & $1,00-2,88$ \\
\hline
\end{tabular}

Me: mediana; M: média; DP: desvio-padrão.

"resolução de problemas" $(M=2,06)$ e "fuga e esquiva" $(M=2,05)$ foram os mais utilizados pelos participantes, seguidos por "reavaliação positiva" $(M=1,95)$. O fator menos utilizado foi o de "afastamento" $(M=0,67)$.

Os resultados da análise estatística dos indicadores de stress em comparação à média da variável renda econômica (em reais) apontaram que os participantes com presença de stress apresentaram médias significativamente superiores $(p=0,02)$ de renda familiar ( $M=955)$, quando comparados aos indivíduos que não apresentaram manifestações de stress $(M=515)$.

A análise dos resultados do Inventário de Estratégias de Coping, em função da presença e ausência de indicadores de stress, apontou que a média obtida em relação ao fator "aceitação de responsabilidade" foi significativamente superior $(p=0,04)$ para os cuidadores com presença de stress $(M=1,14)$, quando comparados com os participantes com ausência de manifestações de stress $(M=0,7)$. Para as demais estratégias de enfrentamento, não foram encontradas diferenças estatisticamente significativas em relação aos indicadores de stress.

A partir da análise das médias dos escores obtidos em relação a cada um dos fatores de enfrentamento em função da variável praticante de religião, observou-se que foi encontrada diferença estatisticamente significativa $(p=0,003)$ entre o maior uso da estratégia de enfrentamento "aceitação de respon- sabilidade" e não ser praticante de religião $(M=1,38)$, quando comparado a ser praticante de religião $(M=0,65)$.

A análise de conteúdo das entrevistas relativas às vivências afetivas dos cuidadores frente ao adoecimento da criança foi agrupada em quatro categorias, a saber: sofrimento/dor; enfrentamento; alterações emocionais; autoexigência. Para melhor descrevê-las, serão utilizados exemplos retirados da transcrição literal das entrevistas.

a) Sofrimento/dor: Diante do adoecimento da criança, os cuidadores relataram a experiência de sofrimento e dor, que ocorreu de forma característica em diferentes momentos. Observou-se que muitos cuidadores apontaram o momento da descoberta da doença como uma situação inesperada:

- Participante 17: "No começo, nossa! Não tava esperando aquilo, foi de repente, né?! Foi do dia pra noite ... que a gente descobriu o problema dele, né?! Aí a gente ficou muito sentido, nossa! Sofreu demais".

Apontaram também para o impacto do início do tratamento:

- Participante 1: "Assim, pra mim no início do tratamento dela foi péssimo, foi péssimo. Fiquei magoada, revoltada. Foi péssimo, péssimo. Émuito mais duro dever ela passando poraquilo. Eu cheguei a falar comigo mesmo que podia ser comigo e não com ela. Não aceitava essa doença".

Descreveram suas vivências frente ao tratamento e as dificuldades deste momento:

- Participante 19: "É um tratamento cansativo, difícil pra qualquer mãe, qualquer pai, difícil. Não é fácil não. Cêtem que ter muita força, muita fé,jávi tanta criança morrer, tanta mãe perder seu filho".

Refletiram sobre a vivência de sofrimento e dor diante da possibilidade de morte:

- Participante 3: "Medo denão ter cura, fiquei muito preocupada com isso. Como éque tava o problema dela, o que ela tava sentindo, achava que era um câncere quenão tinha cura, foi o que eu pensei".

b) Enfrentamento: Os cuidadores relataram que houve um enfrentamento da situação de diversas maneiras. Uma delas foi a crença em Deus, que se manifestava de duas diferentes formas: tendo fé em Deus, mas também responsabilizando-o, ou seja, entregando a situação à vontade de Deus. 


\section{Fé em Deus}

- Participante 2: "O que me ajudou foi a minha fé. Eu tenho muita fé em Deus, me apeguei muito a Deus e se não fosse isso eu não taria do jeito que eu tô hoje não, de maneira alguma".

\section{Responsabilizando Deus}

- Participante 7: "Se Deus não fez de outro jeito.... A vida não é do jeito que a gente quer. Se Deus não quiser que caia uma folha, aquela folha não vai cair, né?! Então eu acredito quevaiser assim, do jeito que Deus quiser. Com esse tratamento todo, eu aprendi a acreditar na vida e acreditar na morte também. Porque eu acredito assim que Deus vai me dar ele atéquando ele achar que eu sou suficientemente capaz de cuidar dele. Na hora que ele falar, ele vaidizer assim: 'Agora ele já concluiu o destino dele, agora ele vai vir para perto de mim.' Eu vou aceitar também. Então, eu não me desespero, não me descontrolo, não me abalo".

Apontaram também para o enfrentamento por meio da adesão ao tratamento e à orientação médica:

- Participante 3: "Aíos médicos chamou, conversou direitinho, falou para mim que tinha cura, que tinha chance de cura, que tinha que tomar os remédios todos direitinhos, tinha as quimio para fazer, eu procurei fazer tudo certinho. Aí, eu falei: 'Se eles falô assim, eu vou segui, né?'. Aíeu seguiné, fiz as quimio tudo certinho, que os médicos pediu para fazer".

Usaram, também, da negação como maneira de enfrentar:

- Participante 10: "Láno fundo vocênão se conforma, né? Eu não me conformo atéhoje. Às vezes eu acordo de manhã eu pego efalo: 'Meu filho não está doente, esse povo tá tudo louco.' Conformar, conformar não. Eu acho que ninguém consegue se conformar".

Em alguns momentos buscaram enfrentar a situação comparando a doença da criança com outras situações que encontravam no hospital:

- Participante 12: "Então aí você começa a se deparar com outras pessoas, ver outras situações. Eu nem sei se é pecado isso, mas você começa pegarforça nisso, aí que você vê que na sua filha não é tão grave como no outro, na sua filha tem jeito, tem tratamento, de $80 \%$ a $90 \%$ de chance de cura. Aívocêcomeça aver do lado as pessoas que já estão desenganadas em relação ao filho deles. Então, aí vocêcomeça a criarforça".

Muitos cuidadores denominaram um dos momentos do tratamento, a manutenção, como fim do tratamento, apontando para uma maior facilidade em enfrentar e vivenciar a situação:

- Participante 3: "Agora que está na manutenção, agora ela tá bem, graças a Deus. Agora o mais difícil já passou".

Apresentaram, também, expectativas positivas como forma de encarar a situação:

- Participante 16: "Mas, eu encarei de uma forma assim: mais tranquila, né?! Porque a gente tem que sempre acreditar que vai dar tudo certo".

c) Alterações emocionais: Diante da situação de adoecimento da criança, os cuidadores vivenciaramna com alterações emocionais:

- Participante 19: "Eu entrei em choque, fiquei muito magra, cheguei a pesar 38 quilos, meio em depressão, fiquei muito ruim".

d) Autoexigência: A partir dos relatos dos cuidadores torna-se perceptível o caráter de autoexigência que a situação acaba impondo:

- Participante 5: ".. eu estava enxergando apenas o meu trabalho, só o meu trabalho. Então isso criou um sentimento de culpa muito grande, por não ter percebido, por ter demorado para tomar uma atitude, levar ele no médico, tal".

\section{Discussão}

Metade dos participantes do estudo (50\%) apresentou indicadores de presença de stress, sendo que a maioria destes (60\%) encontrava-se na fase de resistência. Esses dados apontam para a presença de stress já instalado; esses acompanhantes se encontravam com menores ou esgotados recursos para lidarem com a exigência da situação. Segundo Selye (1983), a adaptabilidade do corpo é finita; assim, pode-se supor um desgaste desses cuidadores, que tiveram que despender atenção a outro (o filho), mas é evidente a necessidade de cuidados também para si. A sintomatologia apresentada pelos participantes, com manifestações de stress, foi $90 \%$ de ordem psicológica, indicando que os 
cuidadores pudessem estar experimentando sentimentos como irritação, depressão, ansiedade e ainda desvalorização pessoal, hipersensibilidade emotiva e pouca confiança em si mesmos. Através da análise qualitativa das entrevistas verificou-se a presença de alterações emocionais em muitos cuidadores. O relato apresentado aponta para manifestações depressivas na vivência da situação. Assim, cabe ressaltar que esta sintomatologia pode influenciar negativamente tanto a qualidade de vida desses cuidadores, quanto a capacidade de oferecer acolhimento e responder às demandas da criança em tratamento, podendo prejudicar o enfrentamento destas à situação.

Com base na análise das entrevistas realizadas, a vivência mais frequentemente identificada diante da situação de adoecimento da criança foi a de sofrimento e dor, que ocorreram nos diferentes momentos do tratamento. Vários estudos realizados com acompanhantes e familiares de pacientes com câncer apontam para o desgaste emocional da vivência da situação em diferentes momentos da doença (Kazak et al., 2004; Kazak et al., 2005; Patiño-Fernandez et al., 2008).

A descoberta da doença foi vivida com muita surpresa pelos cuidadores. Tal dado é semelhante ao descrito por Patterson et al. (2004), que relataram que o diagnóstico do câncer infantil repercute de forma devastadora na família. Muitas vezes ameaça a função diária normal, sendo os familiares forçados a se incumbirem de novos papéis para os quais podem não estar preparados ou querendo aceitar (Northouse \& Peters-Golden, 1993). Nesse mesmo sentido, Bradford (1997) aponta que as necessidades exigidas pela situação de adoecimento e tratamento, tais como: repetidas visitas a médicos, hospitais e a necessidade de assegurar que as medicações sejam dadas em horas específicas, são fatores que potencializam o stress de pais cujos filhos estejam acometidos por alguma doença crônica. No contato com os pais participantes da pesquisa, exigências da situação de tratamento foram muitas vezes apontadas como fatores que dificultam a vivência e acompanhamento da criança. Pode-se pensar que, somado à sobrecarga emocional, os pais precisam assegurar, além de acolhimento à criança, cuidados técnicos e práticos relacionados ao tratamento, aumentando as exigências e provavelmente potencializando as manifestações de 18 stress.
Outro fator associado ao desencadear de sofrimento frente ao diagnóstico de câncer é a existência de preconceitos negativos ligados à doença (Ulisses de Carvalho, 2008; Yin \& Twinn, 2004). Na mesma direção, a confirmação do diagnóstico parece configurar um estopim para o início de pensamentos negativos. Verificou-se, por meio da análise qualitativa, a percepção e conscientização da possibilidade de morte da criança, que acabam por facilitar desestruturações pessoais e manifestações de stress.

Analisando os resultados relativos às manifestações de stress e a variável renda, observou-se que os participantes com presença de stress apresentaram médias significativamente superiores de renda familiar. A renda familiar está relacionada a maiores possibilidades de acesso a serviços de saúde particulares, bem como a informações, o que pode levar a uma postura mais questionadora diante do tratamento, podendo desencadear uma busca incessante por cuidados e maiores exigências internas, gerando maior dificuldade para se adaptar à situação de doença vivenciada. Novos estudos com foco nestas variáveis poderiam elucidar melhor esta relação.

Em relação ao resultado do Inventário de Estratégias de Enfrentamento, verificou-se que as estratégias de enfrentamento"resolução de problemas" $(M=2,06)$ e "fuga e esquiva" $(M=2,05)$ foram as mais utilizadas pelos participantes. Cabe ressaltar que o uso da estratégia "resolução de problemas" refere-se a esforços na direção de solucionar a situação problemática vivida e caracteriza-se como uma estratégia centrada no problema. No entanto, a estratégia de enfrentamento "fuga e esquiva", centrada na emoção, indica uma tentativa de negar a situação vivida. Fayram e Christensen (1995) apontam que estratégias centradas no problema são consideradas adaptativas, buscando remover ou abrandar a fonte estressora, levando em conta a realidade. Já as estratégias de enfrentamento centradas na emoção são apontadas pelos autores como tentativa de substituir ou regular o impacto emocional do stress no indivíduo, originando-se principalmente de processos defensivos, fazendo com que as pessoas evitem confrontar conscientemente a realidade que as ameaça. A partir da análise de conteúdo das entrevistas também foi possível identificar estratégias de enfrentamento focadas no problema como, por exemplo, adesão ao tratamento, 
seguir orientação médica; e focadas na emoção, como o caso da negação da situação.

Foram encontradas diferenças estatisticamente significativas entre a utilização da estratégia de enfrentamento "aceitação de responsabilidade" e maiores indicadores de manifestações de stress. Cabe ressaltar que tal estratégia refere-se à atitude de se culpar frente à situação de adoecimento da criança. Neste sentido, pode-se supor que sentir-se responsável pelo adoecimento do filho influencia negativamente os cuidadores, podendo atuar como um elemento estressor e desencadear maiores índices de manifestações de stress. Menezes et al. (2007) ressalta que, diante da frustração desse momento de dor e incertezas, a família sente-se culpada, como se tivesse falhado na responsabilidade de proteger. Cabe ressaltar que se o "culpar-se" foi um dos aspectos presentes no relato de cuidadores na categoria autoexigência, pode-se supor que a presença do sentimento de culpa acarrete uma sobrecarga emocional adicional ao que a doença, por si só, já ocasiona.

Os participantes não praticantes de religião apresentaram diferença estatisticamente significativa quanto ao maior uso da estratégia "aceitação de responsabilidade", quando comparados aos praticantes de religião. Conforme apontado anteriormente, tal estratégia referese à atitude de se culpar frente à situação de adoecimento da criança. Assim, pode-se postular que a possibilidade de dar sentido ao surgimento da doença como um desígnio de Deus, por exemplo, diminui o sentimento de culpa frente à situação. A análise qualitativa confirma este dado, uma vez que a maioria dos participantes apontou a religiosidade como muito presente para o enfrentamento da situação. Pode-se pensar que a crença e a espiritualidade, de uma forma geral, possibilitam dar sentido à vida e aos acontecimentos presentes nela, diminuindo os conflitos que surgem diante de uma situação inesperada e de sofrimento. Um trabalho escrito por Sapolsky (1994) corrobora os dados encontrados neste estudo. O autor aponta que, por meio de testes endocrinológicos, verificou-se que cuidadores de crianças com câncer em estado terminal apresentaram menores índices de liberação de glucocorticoides, ou seja, menores níveis de stress, quando encontraram na religiosidade a possibilidade de dar sentido à ocorrência da doença. Klassmann, Kochia,
Furukawa, Higarashi e Marcon (2008) apontam, em estudo sobre a experiência de mães de crianças com leucemia, que os cuidadores têm na religiosidade um alívio para o sofrimento ocasionado pelas enfermidades, buscando nela forças para enfrentar a situação e continuar cuidando.

\section{Considerações Finais}

Cabe ressaltar como limites deste estudo a variabilidade da idade das crianças e o número de acompanhantes estudados. Por se tratar de coleta de dados no local de tratamento, sem que a pesquisadora tivesse qualquer interferência na rotina da unidade, trabalhou-se com a faixa etária recebida pelo serviço e com os casos que estavam sendo acompanhados no período da coleta de dados.

O desenvolvimento deste trabalho traz subsídios para o aperfeiçoamento do cuidado aos acompanhantes, uma vez que revelou a presença de stress e também o relato de um importante sofrimento diante do adoecimento da criança nas diferentes fases do tratamento oncológico. A análise dos dados aponta para uma grande exigência e dificuldade na atividade do cuidar, revelando a necessidade de uma rede de suporte a esses cuidadores, com estratégias formais e informais, o que pode vir a diminuir a sobrecarga do cuidador.

Neste contexto de cuidado ao cuidador de crianças com câncer, fazem-se necessários novos estudos que acompanhem o cuidador ao longo das diferentes etapas, com mais recursos de observação do processo. Infere-se a possibilidade de desenvolver habilidades nos indivíduos de modo a melhorar o manejo da situação estressora, objetivando uma melhor qualidade de vida para o cuidador, sua família e a criança doente. Considera-se, ainda, que uma utilização sistemática desses instrumentos no contexto de uma unidade de oncologia pediátrica poderia minimizar a ocorrência de stress, favorecendo intervenções conjuntas da psicologia e da medicina.

\section{Referências}

Bardin, L. (1979). Análise de conteúdo. Lisboa: Edições 70.

Bradford, R. (1997). Children, families and chronic disease. London: Routledge. 
Brasil. Ministério da Saúde. (2008). Instituto Nacional do Câncer. Recuperada em maio, 2008, disponível em http://www.inca.org.br/cancer

Fayram, E. S., \& Christensen, P. J. (1995). Planning: strategies and nursing orders. In P. J. Christensen \& J. W. Kenney. Nursing process: application of conceptual models (pp.164-185). St. Louis: Mosby.

Françoso, L. P. C., \& Valle, E. R. M. (1999). A Criança com câncer: estudo preliminar. Pediatria Moderna, 35 (5), 320-331.

Guido, L. A. (2003). Stress e coping entre enfermeiros de centro cirúrgico e recuperação anestésica. Tese de doutorado não-publicada, Escola de Enfermagem, Universidade de São Paulo.

Kazak, A. E., Alderfer, M., Rourke, M. T., Simms, S., Streisand, R., \& Grossman, J. R. (2004). Posttraumatic stress disorder (PTSD) and posttraumatic stress symptoms (PTSS) in families of adolescent childhood cancer survivors. Journal of Pediatric Psychology, 29 (3), 211-219.

Kazak, A. E., Boeving, C. A., Alderfer, M., Hwang, W-T, \& Reilly, A. (2005). Posttraumatic stress symptoms during treatment in parents of children with cancer. Journal Clinical Oncology, 23 (30),7405-7410.

Klassmann, J., Kochia, K. R. A, Furukawa, T. S., Higarashi, I. H., \& Marcon, S. S. (2008). Experiência de mães de crianças com leucemia: sentimentos acerca do cuidado domiciliar. Revista da Escola de Enfermagem USP, 42 (2), $321-330$

Knafl, K. A., \& Deatrick, J. A. (2003). Further refinement of the family management style framework. Journal of Family Nursing, 9 (3), 232-56.

Lazarus, R. S., \& Folkman, S. (1984). Stress, appraisal and coping. New York: Sringer Publishing Company.

Lipp, M. E. N. (1996). Stress: conceitos básicos. In M. E. N. Lipp (Ed.), Pesquisas sobrestress no Brasil: saúde, ocupações e grupos de risco (pp.17-31). São Paulo: Papirus.

Lipp, M. E. N. (2000). Manual do Inventário de Sintomas de Stress para Adultos de Lipp. São Paulo: Casa do Psicólogo.

Lipp, M. E. N., \& Malagris, L. N. (1998). Manejo do estresse. In: B. Rangè (Org.), Psicoterapia comportamental e cognitiva: pesquisa, prática, aplicações e problemas (pp.279-292). São Paulo: Editora PSY.
Menezes, C. N. B., Passareli., P. M., Drude, F. S., Santos, M. A., \& Valle, E. R. M. (2007). Câncer infantil: organização familiar e doença. Revista Mal Estar e Subjetividade, 7 (1), 191-210.

Northouse, L. L., \& Peters-Golden, H. (1993). Cancer and the family: strategies to assist spouses. Seminars in Oncology Nursing, 9 (2), 74-82.

Patterson, J. M., Holm, E. K., \& Gurney, J. G. (2004). The impact of childhood cancer on the family: a qualitative analysis of strains, resources, and coping behaviors. Psycho-Oncology, 13 (6), 390-407.

Patiño-Fernandez, A. M., Pai, A. L. H., Alderfer, M., Hwang, W-T., Reilly, A., \& Kazak, A. E. (2008). Acute stress in parents of children newly diagnosed with cancer. Pediatric Blood \& Cancer, 50 (2), 289-292.

Savoia, M. G. (1999). Escala de eventos vitais e de estratégias de enfrentamento (Coping). Revista de Psiquiatria Clínica, 26 (2), 57-67.

Sapolsky, R. M. (1994). Why zebras don't get ulcers: a guide to stress, stress-related diseases, and coping. New York: W.H. Freeman and Company.

Selye, H. (1956). The stress of life. New York: Longmans.

Selye, H. (1983). The Stress Concept: past, present and future. In C. L. Cooper (Ed.), Stress reserarch: issues for the eighties (pp.1-20). New York: John Wiley and Sons.

Ulysses de Carvalho, C. S. (2008). A necessária atenção à família do paciente oncológico. Revista Brasileira de Cancerologia, 54 (1), 97-102.

Valle, E. R. M., \& Françoso, L. P. C. (1999). Câncer infantil: algumas informações. In E. R. M. Valle \& L. C. Françoso. Psico-oncologia pediátrica: vivências da criança com câncer (pp.15-18). Ribeirão Preto: Scala.

Yin L. K., \& Twinn S. (2004) The effect of childhood cancer on Hong Kong Chinese families at different stages of the disease. Cancer Nursing, 27 (1),17-24.

Recebido em: 7/4/2008

Versão final reapresentada em: 23/9/2008

Aprovado em: 30/10/2008 